\title{
ON A THEOREM BY A. E. TAYLOR
}

SHAUL R. FOGUEL

Let $B$ be a complex normed linear space. It is well known [1] that, for any bounded linear functional $\phi$ defined on a linear subspace $M$ of $B$, there exists a norm-preserving linear extension $f$ of $\phi$ to $B$, i.e. a bounded linear functional $f$ defined on $B$ such that (i) $f(x)$ $=\phi(x)$ for all $x \in M$, (ii) $\|f\|_{B}=\|\phi\|_{M}$, where $\|f\|_{B}$ and $\|\phi\|_{M}$ denote the norms of bounded linear functionals $f$ and $\phi$ on $B$ and $M$, respectively. It was proved by A. E. Taylor [2] that if the conjugate space of $B$ is strictly convex, then $f$ is uniquely determined by $\phi$. The purpose of this note is to show that the converse of this theorem is true, i.e. we want to prove the following

TheOREM. Let $B$ be a complex normed linear space whose conjugate space is not strictly convex. Then there exists a bounded linear functional defined on a linear subspace of $B$ for which a norm preserving linear extension to $B$ is not unique.

Proof. Let $f_{1}$ and $f_{2}$ be two bounded linear functionals on $B$ such that (i) $f_{1} \neq f_{2}$, (ii) $\left\|f_{1}\right\|_{B}=\left\|f_{2}\right\|_{B}=\left\|\left(f_{1}+f_{2}\right) / 2\right\|_{B}=1$. Let us put $M$ $=\left\{x \mid f_{1}(x)=f_{2}(x)\right\}$ and $\phi(x)=f_{1}(x)=f_{2}(x)$ on $M$. It suffices to prove that $\|\phi\|_{M}=1$. Let $z$ be an element of $B$ such that $f_{1}(z)-f_{2}(z)=1$. Then every element $x$ of $B$ can be uniquely expressed in the form: $x=y+a z$, where $y \in M$ and $a=f_{1}(x)-f_{2}(x)$ is a complex number. Let $\left\{x_{n} \mid n=1,2, \cdots\right\}$ be a sequence of elements of $B$ such that $\left\|x_{n}\right\|=1$ for $n=1,2, \cdots$ and $\lim _{n \rightarrow \infty}\left(f_{1}\left(x_{n}\right)+f_{2}\left(x_{n}\right)\right) / 2=1$. Then it is easy to see that $\lim _{n \rightarrow \infty} f_{1}\left(x_{n}\right)=\lim _{n \rightarrow \infty} f_{2}\left(x_{n}\right)=1$. Thus, if we put $x_{n}=y_{n}+a_{n} z$, where $y_{n} \in M$ and $a_{n}=f_{1}\left(x_{n}\right)-f_{2}\left(x_{n}\right), n=1,2, \cdots$, then $\lim _{n \rightarrow \infty} a_{n}=0$, and hence

$$
\lim _{n \rightarrow \infty}\left\|y_{n}\right\|=\lim _{n \rightarrow \infty}\left\|x_{n}\right\|=1 \text { and } \lim _{n \rightarrow \infty} \phi\left(y_{n}\right)=\lim _{n \rightarrow \infty} f_{1}\left(y_{n}\right)=1 .
$$

From this follows that $\|\phi\|_{M} \geqq 1$ and hence $\|\phi\|_{M}=1$.

\section{REFERENCES}

1. H. F. Bohnenblust and A. Sobczyk, Extension of functionals on complex linear spaces, Bull. Amer. Math. Soc. vol. 44 (1938) pp. 91-93.

2. A. E. Taylor, The extension of linear functionals, Duke Math. J. vol. 5 (1939) pp. 538-547.

University of Pennsylvania

Received by the editors January 30, 1956 and, in revised form, November 30, 1956. 